\title{
Smoking prevalence and seizure control in Chinese males with epilepsy.
}

\author{
Hui Gao, Josemir W. Sander , Xudong Du, Jiani Chen, Cairong Zhu, Dong Zhou
}

\begin{abstract}
Smoking has negative effect on most diseases, yet it is under-investigated in people with epilepsy; thus its role is not clear in the general population with epilepsy. We performed a retrospective pilot study on males with epilepsy to determine the smoking rate and its relationship with seizure control using univariate analysis to calculate odds ratios (ORs) and also used a multi-variate logistic regression model., The smoking rate in our sample of 278 individuals was $25.5 \%$, which is lower than the general Chinese population smoking rate amongst males of $52.1 \%$. We used two classifications: the first classified epilepsy as generalized, or by presumed topographic origin (temporal, frontal, parietal and occipital). the second classified the individuals dominant seizure type as generalized tonic clonic seizure (GTCS), myoclonic seizure (MS), complexed partial seizure (CPS), simple partial seizure (SPS), and secondary GTCS (sGTCS). The univariable analysis of satisfactory seizure control profile and smoking rate in both classifications showed a trend towards a beneficial effect of smoking although most were not statistically significant. Considering medication is an important confounding factor that would largely influence seizure control, we also conducted multi-variable analysis for both classifications with drug numbers and dosage. The result of our model also suggested that smoking is a protective factor. Our findings seem to suggest that smoking could have a potential role in seizure control although confounders need exploration particularly in view of the potential long term health effects. Replication in a much larger sample is needed as well as case control studies to elucidate this issue.
\end{abstract}

Key words: Smoking, Epilepsy, seizure control

\section{Introduction}

Cigarette smoking is a worldwide health problem. Given its high prevalence among the population, many studies have been done over the years regarding its health effects. In some diseases, smoking has negative effect, while in others, for example in inflammatory bowel diseases ${ }^{1,2}$, it can be a protective factor.

Some aspects of smoking in people with epilepsy have been investigated. Most studies 
considered the general prevalence rate of smoking in people with epilepsy rather than the effect of smoking ${ }^{3-8}$. In several studies the smoking rates in people with epilepsy were always higher than in the general population, with rates ranging from $21.8 \%$ to $38.8 \%{ }^{3-8}$; the smoking rate in people with epilepsy is also higher than in most other chronic diseases ${ }^{6}$. The smoking rate was even higher in people with drug resistant epilepsy ${ }^{9}$. A recent study, however, showed the rate was dropping ${ }^{10}$.

Some reports suggest that smoking can have negative effects on seizures and epilepsy (ref.). There are studies concluding that children of women who smoke have a higher rate of febrile seizures ${ }^{11-13}$, although other studies did not find confirm this ${ }^{14,15}$. Women with epilepsy have been shown to have an increased risk of premature contractions and premature birth ${ }^{16,17}$. Self-reporting seizure triggering factors suggest that smoking may be a trigger factor with other factors such as medication withdrawn and sleep deprivation ${ }^{18}$. Poor adherence to a medication regime may co-occur in adolescents ${ }^{19}$. In a prospective study of a nurse cohort followed for more than 20 years, current cigarette smoking was associated with an increased risk of seizures (RR 2.60, 95\% CI 1.53-4.42), and past smoking was associated with an increased risk of epilepsy (RR 1.46, 95\% CI 1.01-2.12) ${ }^{20}$. Smoking may also influence drug concentrations in serum. Several studies suggest that smokers may have lower serum drug concentrations than non-smokers while taking the same drug (such as lamotrigine (LTG)) due to smoking related hepatic enzyme changes ${ }^{21}$. Nicotine, a main component in cigarettes, has also been shown to lower the seizure threshold in animals ${ }^{22}$.

Conversely, nicotine has been confirmed as being protective and as having a treatment effect in rare hereditary epilepsy (autosomal dominant nocturnal frontal lobe epilepsy [ADNFLE]). The mutated gene in ADNFLE is related to functioning of the nicotinic acetylcholine receptor. People with epilepsy in families with ADNFLE who smoked or used nicotine patches had a better overall seizure control than those who do not ${ }^{23}$. However, in sporadic nocturnal frontal lobe epilepsy failed to determine the role of smoking ${ }^{24}$. Another study showed that nicotine patch use in video EEG monitoring increased the hospitalization of people with epilepsy as fewer seizure events were captured $^{25}$. Thus there is some circumstantial evidence for smoking and nicotine to have a beneficial effect on seizure control.

Data from 2015, suggest that the general smoking rate in the Chinese population is $27.7 \%,(52.1 \% \text { in males and } 2.7 \% \text { in females })^{26}$. Smoking rates amongst people with 
epilepsy in China has not been recently ascertained. We aimed to investigate the role of smoking in people with epilepsy in China.

\section{Materials and methods}

We have an established clinical databank for people with epilepsy in our center. The data bank should include data on all those who routinely attend Data includes contact information and demographics such as name, sex, age, education profile, marriage status and number of children. Recorded clinical data includes diagnosis, EEG, neuroimaging, seizure types, current seizure frequency, medication profile and epilepsy phase (table 1).

Table 1. Definition of different phases of an individual with epilepsy

\begin{tabular}{|c|c|}
\hline Active phase & $\begin{array}{l}\text { Individuals have had a seizure within the } \\
\text { previous year and are changing their } \\
\text { medication }\end{array}$ \\
\hline Maintenance phase & $\begin{array}{l}\text { a. Seizures have stopped and no } \\
\text { medication changes have taken place } \\
\text { within the previous year (Type 1) } \\
\text { b. Individuals have had a seizure within } \\
\text { the previous year but have had no } \\
\text { medication change (Type 2) }\end{array}$ \\
\hline Medication reducing phase & Individuals reducing their medications \\
\hline No medication phase & $\begin{array}{l}\text { Individuals who have reduced and } \\
\text { subsequently stopped their medications }\end{array}$ \\
\hline
\end{tabular}

The establishment of the databank was approved by West China Hospital Ethics Committee and written consent is always provided by individuals before data collected. In November 2015, we contacted by telephone males in the databank inquiring about their smoking status (as below). Only those in the databank prior to November 2014 were contacted as to avoid including individuals with newly diagnosed epilepsy. Only males were included in this pilot study as most smokers in China are males. We excluded people with a history of poor adherence and those whose final diagnosis was not epilepsy. We also excluded newly diagnosed patients (hard to evaluate their seizure 
control in a short time) and symptomatic epilepsy.

We collected data on smoking status as - never-smoker, current smoker (at least 1 cigarette per day in past 6 months), former smoker (smoking stopped by the time of the study). For smokers and former smokers were recorded number of years smoking. We also performed the Fagerström Test of Nicotine Dependence (FTND) on the current smokers ${ }^{27}$. This includes 6 questions, and we calculated the nicotine dependence level of individuals by adding the grade of all items together. Smokers with a total grade lower than 6 were considered as having a low to moderate nicotine dependence level and those with grade equal or higher than 6 were considered as having a high nicotine dependence level.

\subsection{Epilepsy classification}

Individuals were classified based on clinical judgement as having focal or generalized epilepsy, and by the dominant seizure type. Focal or generalized epilepsy included categories such as temporal lobe epilepsy, frontal lobe epilepsy, parietal lobe epilepsy, occipital lobe epilepsy and generalized epilepsy. For dominant seizure types, individuals were grouped as having generalized tonic clonic seizures (GTCS), myoclonic seizures (MS), simple partial seizures (SPS), complexed partial seizures (CPS) and secondarily generalized tonic clonic seizure (sGTCS).

\subsection{Smoking rate and seizure control}

For simplicity, we classified seizure control profile into satisfactory seizure control and unsatisfactory seizure control. We excluded individuals in the Type 2 maintenance phase who had seizures within a year without medication change because the reasons for not changing their medication were complicated. They may be less affected by their seizures and decided not to increase dose of medication or they could have drug resistant epilepsy or they may be having seizures frequently but refused to take more medication for worrying side effects. These two situations couldn't be considered neither as satisfied nor unsatisfied seizure control. So satisfactory seizure control was defined as type 1 maintenance phase, reduced medication phase and no medication. Unsatisfactory seizure control was defined as being in the active phase.

All our data was processed in SAS version 9.4 (SAS Institute, Cary, NC, USA). The smoking rate was calculated based on the whole cohort and both classifications. For 
different categories within a classification, we also compared the difference of smoking rate by chi-square to establish whether smoking rate is different between categories.

The seizure control profile under each category of classification was compared by calculation of odds ratios [OR]). For example, in temporal lobe epilepsy using the focal or generalized epilepsy classification, we calculated the number of individuals who smoke with satisfactory seizure control, those who smoke with unsatisfactory seizure control, those who do not smoke with satisfactory seizure control and those who do not smoke with unsatisfactory seizure control, thus we can generate an OR and also identify its statistical significance. We did the same for all categories in 2 different classification systems.

\subsection{Multi-variable analysis for smoking and epilepsy}

The control profile in an individual is primarily based on anti-epileptic drugs (AEDs), thus medications were considered as confounding factors in our analysis. We simply categorized the number of medications as equal or less than 2 medications and more than 2 medications. Furthermore, we considered comparative dosage of their medications as small or large dosage using definition of daily drug dosage (DDD) among different AEDs by WHO.

Table 2. Definition of small and large dosage for a patient based on medication number and dosage for each medication

\begin{tabular}{|c|c|c|}
\hline & Small dosage & Large dosage \\
\hline Only 1 medication & $\leq 50 \% \mathrm{DDD}$ & $>50 \% \mathrm{DDD}$ \\
\hline \multirow[t]{2}{*}{2 medications } & a. Both $\leq 50 \% \mathrm{DDD}$ & a. Both $>50 \%$ DDD \\
\hline & b. $1 \leq 50 \% \mathrm{DDD}$ & \\
\hline \multirow[t]{3}{*}{3 medications } & a. All $\leq 50 \%$ DDD & a. $\quad 2>50 \% \mathrm{DDD}$ \\
\hline & b. $1>50 \%$ DDD & b. All $>50 \%$ DDD \\
\hline & other $2 \leq 50 \%$ DDD & \\
\hline \multirow[t]{5}{*}{4 medications } & a. All $\leq 50 \% \mathrm{DDD}$ & a. $3>50 \% \mathrm{DDD}$ \\
\hline & b. $1>50 \% \mathrm{DDD}$ & $1 \leq 50 \% \mathrm{DDD}$ \\
\hline & other $\leq 50 \%$ DDD & b. All $>50 \% \mathrm{DDD}$ \\
\hline & c. $2>50 \% \mathrm{DDD}$ & \\
\hline & other $2 \leq 50 \%$ DDD & \\
\hline
\end{tabular}


We performed multiple logistic regression analysis model based on medication numbers, dosage levels, age of the patient (between different age of patients, the smoking rate can be different), their smoking status as yes or no and separately using both classification methods. We calculated the coefficients and evaluated whether smoking is a protective factor for seizure control in people with epilepsy.

\subsection{Dependence level of nicotine and seizure control}

For smokers, we also analyzed their nicotine dependence level as low or high and explored its relationship with seizure control. We performed Chi square test to identify whether the dependence level on nicotine could affect seizure control profile. If the expected frequency bigger than 1 but lower than 5, Yate's correction will be done for the small sample size.

\section{Results}

\subsection{Prevalence of smoking in people with epilepsy}

\section{a. General data of our cohort and smoking prevalence}

Among 1000 individuals we reviewed in our data bank, 342 were males and met our inclusion criteria; 64 were further excluded as we were unable to contact them. Only 2 $(0.72 \%)$ individuals stopped smoking (they all quitted more than 5 years) among the 278 we included in our analysis; $71(25.5 \%)$ were current smokers. Thus the prevalence of smoking among males with epilepsy in our cohort is $25.5 \%$, while among general adult males in China it is $52 \%$.

In our cohort, 70 people $(25.2 \%)$ had temporal lobe epilepsy, $41(14.75 \%)$ had frontal lobe epilepsy, two had parietal lobe epilepsy, one had occipital lobe epilepsy and 164 (59\%) had generalized epilepsy. Regarding the dominant seizure type, 218 (78.4\%) had GTCS, two had MS, 7 had SPS, 42 (15\%) had CPS and nine (3.2\%) had SGTCS. We thus excluded those with parietal lobe epilepsy and occipital lobe epilepsy, and those with MS and SPS, due to their small numbers in our cohort. We simply classified our patients as smoking and not smoking according to their current smoking status.

\section{b. Different smoking rates among people with different types of epilepsy}

Table 3. Smoking rate in focal epilepsies or generalized epilepsy

\begin{tabular}{lllll}
\hline Not smoking & smoking & Total & Smoking rate & Chi-square Test \\
\hline
\end{tabular}




\begin{tabular}{cccccc}
\hline Temporal lobe epilepsy & 40 & 17 & 57 & $29.8 \%$ & \\
Frontal lobe epilepsy & 29 & 6 & 35 & $17.1 \%$ & $\chi^{2}=1.9226$ \\
Generalized epilepsy & 114 & 36 & 150 & $24.0 \%$ & $P=0.38$ \\
\hline Total & 183 & 59 & 242 & $24.4 \%$ & \\
\hline
\end{tabular}

Table 4. Smoking rate according to dominant seizure type

\begin{tabular}{cccccc}
\hline & Not smoking & Smoking & Total & Smoking rate & Chi-square Test \\
\hline GTCS & 147 & 47 & 194 & $24.2 \%$ & \\
CPS & 27 & 7 & 34 & $20.6 \%$ & $\chi^{2}=0.2225$ \\
SGTCS & 7 & 2 & 9 & $22.2 \%$ & $P=0.89$ \\
\hline Total & 181 & 56 & 237 & $23.6 \%$ & \\
\hline
\end{tabular}

The Chi-square test shows no difference in smoking rates among people with different types of epilepsy.

\subsection{Smoking and seizure control}

\section{a. Analysis in different origins of epilepsy}

Table 5. Seizure control and epilepsy in focal epilepsies or generalized epilepsy

\begin{tabular}{ccccccc}
\hline \multirow{2}{*}{$\begin{array}{c}\text { Epilepsy } \\
\text { origin }\end{array}$} & Control & Not & Smoking & Total & OR & 95\% CI of OR \\
\hline Temporal & Unsatisfactory & 23 & 4 & 27 & & \\
lobe & Satisfactory & 17 & 13 & 30 & 4.3971 & $1.2176-15.8792$ \\
Epilepsy & Total & 40 & 17 & 57 & & \\
\cline { 2 - 7 } Frontal & Unsatisfactory & 12 & 1 & 13 & & \\
Lobe & Satisfactory & 17 & 5 & 22 & 3.5294 & $0.3644-34.1847$ \\
Epilepsy & Total & 29 & 6 & 35 & & \\
\cline { 2 - 7 } Generalized & Unsatisfactory & 34 & 8 & 42 & & \\
epilepsy & Satisfactory & 80 & 28 & 108 & 1.4875 & $0.6156-3.5943$ \\
& Total & 114 & 36 & 150 & & \\
\hline
\end{tabular}

The ORs in temporal lobe epilepsy, frontal lobe epilepsy and generalized epilepsy are 
all higher than 1, suggesting a tendency of smoking to be a protective factor in all three types of epilepsy; the $95 \%$ CIs are wide. In temporal lobe epilepsy the lower confidence limit of the OR value is higher than 1 suggesting that, in this group, smoking is a statistically significant protective factor. This analysis, however, does not include confounders, so we performed multi-variable analyses.

Table 6. Multi-variable analysis for control of seizures in focal or generalized epilepsy

\begin{tabular}{|c|c|c|c|c|}
\hline & $\boldsymbol{\beta}$ & $P$ value & OR & $95 \% \mathrm{CI}$ of $\mathrm{OR}$ \\
\hline \multicolumn{5}{|l|}{ Number of drugs } \\
\hline$>2$ & referent & - & - & - \\
\hline$\leqslant 2$ & 1.4788 & 0.0002 & 4.388 & $2.020-9.532$ \\
\hline \multicolumn{5}{|l|}{ Drug dosage } \\
\hline Large & referent & - & - & - \\
\hline Small & 0.5459 & 0.0726 & 1.726 & $0.951-3.132$ \\
\hline \multicolumn{5}{|l|}{ Epilepsy Origin } \\
\hline Temporal lobe epilepsy & referent & & - & - \\
\hline Frontal lobe epilepsy & 0.4141 & 0.3848 & 1.513 & $0.595-3.850$ \\
\hline Generalized epilepsy & 0.7717 & 0.0299 & 2.163 & $1.078-4.341$ \\
\hline \multicolumn{5}{|l|}{ Smoking } \\
\hline No & referent & & - & - \\
\hline Yes & 0.9016 & 0.0174 & 2.463 & $1.172-5.178$ \\
\hline age & 0.00243 & 0.8114 & 1.002 & $0.983-1.023$ \\
\hline
\end{tabular}

For each factor we listed here, the first line is used as a referent in our analysis. The goal is satisfactory seizure control profile. Statistically significant OR>1 means comparing to the referent, the chance of satisfactory seizure control is increased. Statistically significant OR $<1$ means the opposite.

This logistic regression model shows that smoking is a protective factor for seizure control with $\mathrm{OR}=2.463$ ( 95\%CI 1.172-5.178 ). Similar to univariate analysis, smoking is still a protective factor in consideration of different medication profile for patients. In our model, taking fewer medications is also associated with better seizure control. We think that's because fewer drugs mean the seizure respond to medication and is not drug resistant. Except for fewer medication, generalized epilepsy is related to better 
control compared to temporal lobe epilepsy and frontal lobe epilepsy.

Age and drug dosage is not related with seizure control based on our data.

\section{b. Analysis in different dominant types of seizures in epilepsy}

We did the same analysis in regard of different types of seizure as a classification for people with epilepsy.

Table 7. Seizure control and epilepsy in different dominant seizure types

\begin{tabular}{|c|c|c|c|c|c|c|}
\hline $\begin{array}{c}\text { Seizure } \\
\text { type }\end{array}$ & $\begin{array}{l}\text { Control } \\
\text { profile }\end{array}$ & $\begin{array}{c}\text { Not } \\
\text { smoking }\end{array}$ & Smoking & Total & OR & $95 \% \mathrm{CI}$ of OR \\
\hline \multirow{3}{*}{ GTCS } & Unsatisfactory & 53 & 10 & 63 & \multirow{3}{*}{2.0862} & \multirow{3}{*}{$0.9607-4.5302$} \\
\hline & Satisfactory & 94 & 37 & 131 & & \\
\hline & Total & 147 & 47 & 194 & & \\
\hline \multirow{3}{*}{ CPS } & Unsatisfactory & 14 & 3 & 17 & \multirow{3}{*}{1.4359} & \multirow{3}{*}{$0.2685-7.6777$} \\
\hline & Satisfactory & 13 & 4 & 17 & & \\
\hline & Total & 27 & 7 & 34 & & \\
\hline \multirow{3}{*}{ SGTCS } & Unsatisfactory & 0 & 2 & 2 & \multirow{3}{*}{$\begin{array}{c}\text { Not } \\
\text { Applicable }\end{array}$} & \multirow{3}{*}{ Not } \\
\hline & Satisfactory & 2 & 5 & 7 & & \\
\hline & Total & 2 & 7 & 9 & & \\
\hline
\end{tabular}

Table 8. Multi-variable analysis for control profile in dominant seizure type based analyzed epilepsy patients

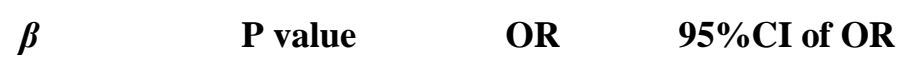

\section{Number of drugs}

$\begin{array}{lrccc}>2 & \text { referent } & - & - & - \\ \leqslant 2 & 1.4918 & 0.0001 & 4.445 & 2.059-9.595\end{array}$

\section{Drug dosage}

$\begin{array}{lcccc}\text { Large } & \text { referent } & - & - & - \\ \text { Small } & 0.5399 & 0.0752 & 1.716 & 0.947-3.110\end{array}$

\section{Dominant seizure}

SGTCS

CPS

referent

$-1.0988$

0.2457

0.333

0.052-2.130 

GTCS
$-0.5678$
0.5177
0.567
$0.101-3.166$

smoking

\begin{tabular}{cccccc} 
& No & referent & & - & - \\
& Yes & 0.8186 & 0.0307 & 2.267 & $1.079-4.764$ \\
age & & -0.00006 & 0.0105 & 1.000 & $0.980-1.021$ \\
\hline
\end{tabular}

The result is similar to the other classification. The univariate analysis still shows a tendency of smoking being a protective factor, but none of the ORs is statistically significant as we showed before, but the multivariate analysis has a significant OR $=2.267(95 \% \mathrm{CI} 1.079-4,764)$ for having protective effect compared to no smoking.

\subsection{Dependence level of nicotine and seizure control in smoking patients}

Table 9. Nicotine dependence level and seizure control

\begin{tabular}{ccccc}
\hline $\begin{array}{c}\text { Control } \\
\text { profile }\end{array}$ & Low dependence & High dependence & Total & Chi-square Test \\
\hline unsatisfied & 8 & 5 & 13 & $\chi_{\mathrm{c}}^{2}=0.0038$ \\
Satisfied & 31 & 15 & 46 & $P=0.9507$ \\
\hline Total & 39 & 20 & 59 & \\
\hline
\end{tabular}

Since we have showed smoking is a protective factor in both classifications and the populations are relatively small, we analyzed all individuals as one group to investigate nicotine dependence level and seizure control; no association was found.

\section{Discussion}

Our findings suggest smoking rate in Chinese adult males with epilepsy is much lower than in the general adult male population. Since our study is a retrospective observational study, our results suggested there is a tendency for smoking and less seizure frequnency.

There seems to be a relatively low smoking rate among people with epilepsy in China particularly in males and this seems to be different scenario from that in other countries in which there seem to be a higher prevalence of smoking amongst people with epilepsy than in the general population. This may be due to public health campaigns over 
emphasizing negative effects of smoking which may led to people assume its negative role in epilepsy. Different from more developed countries, the Chinese patient-doctor mode is still more paternal, which means that people tend to follow their physician's instructions in their daily life. In recent years, people have been more careful about their own health because of better life quality. There could also be reasons such as more opportunities for communication between physicians and patients with all kinds of social media, and the patients are therefore better educated. This gives us more responsibility to inform our patients with more accurate information and more evidence based instruction. It is also possible, particularly in view of the lower prevalence of smoking in people with epilepsy than in the general population, that people with worse seizure control may have previously stopped smoking in an attempt to improve their overall health and, potentially, their seizure control.

Our findings seem to suggest a potential protective role of smoking; the higher dependence level on nicotine, however, is not related to better overall control in those who smoke. This may show that smoking/nicotine does not affect the seizure control profile by its intensity, or that it changes epileptic network in a qualitative way. Whether the change is stable or stops with smoking cession is unknown. Maybe extract from cigarette as adjunctive medication or nicotine therapy could help obtain better seizure control in patients. More studies could also be performed to investigate how smoking affects the whole epilepsy network in the brain to add for more evidence on this topic. We also found a slightly (non-significant) higher rate of smoking among people with temporal lobe epilepsy. This may be related to temporal lobe epilepsy affecting the emotion of patient, thus individuals may be more anxious or depressed, which could be a reason for them to smoke. In this way, the emotional network may also affect the seizure control of an individual, which smoking can act on, and exert its protective effect, as seen in our study.

Our study is just a pilot study, and it was limited by a small number in some subgroups and retrospectively. There should be a better designed, more confounders considered and prospective study to be explored in the future.

\section{Reference}

1. Beaugerie L, Massot N, Carbonnel F, Cattan S, Gendre JP, Cosnes J. Impact of cessation of smoking on the course of ulcerative colitis. The American journal of 
gastroenterology 2001;96:2113-6.

2. Cosnes J, Carbonnel F, Carrat F, Beaugerie L, Cattan S, Gendre J. Effects of current and former cigarette smoking on the clinical course of Crohn's disease. Alimentary pharmacology \& therapeutics 1999;13:1403-11.

3. Cui W, Zack MM, Kobau R, Helmers SL. Health behaviors among people with epilepsy--results from the 2010 National Health Interview Survey. Epilepsy \& behavior : E\&B 2015;44:121-6.

4. Elliott JO, Moore JL, Lu B. Health status and behavioral risk factors among persons with epilepsy in Ohio based on the 2006 Behavioral Risk Factor Surveillance System. Epilepsy \& behavior : E\&B 2008;12:434-44.

5. Ferguson PL, Chiprich J, Smith G, et al. Prevalence of self-reported epilepsy, health care access, and health behaviors among adults in South Carolina. Epilepsy \& behavior : E\&B 2008;13:529-34.

6. Hinnell C, Williams J, Metcalfe A, et al. Health status and health-related behaviors in epilepsy compared to other chronic conditions--a national population-based study. Epilepsia 2010;51:853-61.

7. Kobau R, DiIorio CA, Price PH, et al. Prevalence of epilepsy and health status of adults with epilepsy in Georgia and Tennessee: Behavioral Risk Factor Surveillance System, 2002. Epilepsy \& behavior : E\&B 2004;5:358-66.

8. Torriani O, Vuilleumier F, Perneger T, et al. Epilepsy and tobacco smoking: a crosssectional study. J Neurol 2016.

9. Hamilton M, McLachlan RS, Burneo JG. Can I go out for a smoke? A nursing challenge in the epilepsy monitoring unit. Seizure 2009;18:285-7.

10. Roberts JI, Patten SB, Wiebe S, Hemmelgarn BR, Pringsheim T, Jette N. Healthrelated behaviors and comorbidities in people with epilepsy: Changes in the past decade. Epilepsia 2015;56:1973-81.

11. Vestergaard M, Wisborg K, Henriksen TB, Secher NJ, Ostergaard JR, Olsen J. Prenatal exposure to cigarettes, alcohol, and coffee and the risk for febrile seizures. Pediatrics 2005;116:1089-94.

12. Berg AT, Shinnar S, Shapiro ED, Salomon ME, Crain EF, Hauser WA. Risk factors for a first febrile seizure: a matched case-control study. Epilepsia 1995;36:334-41.

13. Nelson KB, Ellenberg JH. Prenatal and perinatal antecedents of febrile seizures. Ann Neurol 1990;27:127-31. 
14. Vahidnia F, Eskenazi B, Jewell N. Maternal smoking, alcohol drinking, and febrile convulsion. Seizure 2008;17:320-6.

15. Asadi-Pooya AA, Hojabri K. Risk factors for childhood epilepsy: a case-control study. Epilepsy \& behavior : E\&B 2005;6:203-6.

16. Harden CL, Hopp J, Ting TY, et al. Management issues for women with epilepsyFocus on pregnancy (an evidence-based review): I. Obstetrical complications and change in seizure frequency: Report of the Quality Standards Subcommittee and Therapeutics and Technology Assessment Subcommittee of the American Academy of Neurology and the American Epilepsy Society. Epilepsia 2009;50:1229-36.

17. Hvas CL, Henriksen TB, Ostergaard JR, Dam M. Epilepsy and pregnancy: effect of antiepileptic drugs and lifestyle on birthweight. Bjog 2000;107:896-902.

18. Balamurugan E, Aggarwal M, Lamba A, Dang N, Tripathi M. Perceived trigger factors of seizures in persons with epilepsy. Seizure 2013;22:743-7.

19. Kyngas H. Compliance with health regimens of adolescents with epilepsy. Seizure 2000;9:598-604.

20. Dworetzky BA, Bromfield EB, Townsend MK, Kang JH. A prospective study of smoking, caffeine, and alcohol as risk factors for seizures or epilepsy in young adult women: data from the Nurses' Health Study II. Epilepsia 2010;51:198-205.

21. Reinsberger C, Dorn T, Kramer G. Smoking reduces serum levels of lamotrigine. Seizure 2008;17:651-3.

22. Sood N, Hota D, Sahai AK, Chakrabarti A. Nicotine reversal of anticonvulsant action of topiramate in kainic acid-induced seizure model in mice. Nicotine Tob Res 2011;13:1084-91.

23. Brodtkorb E, Picard F. Tobacco habits modulate autosomal dominant nocturnal frontal lobe epilepsy. Epilepsy \& behavior : E\&B 2006;9:515-20.

24. Naldi I, Bisulli F, Vignatelli L, et al. Tobacco habits in nocturnal frontal lobe epilepsy. Epilepsy \& behavior : E\&B 2013;26:114-7.

25. Parikh MS, Haltiner AM, de Menezes MS, et al. Nicotine patches--do they influence seizure number, stay durations or seizure rate during video EEG telemetry? Epilepsy \& behavior : E\&B 2012;23:389-91.

26. Report of cigarette consumption of adult Chinese in 2015 Shanghai Journal of Preventive Medicine, 2015;12.

27. Heatherton TF, Kozlowski LT, Frecker RC, Fagerstrom KO. The Fagerstrom Test 
for Nicotine Dependence: a revision of the Fagerstrom Tolerance Questionnaire. British journal of addiction 1991;86:1119-27.

Appendix

FTND for current smokers

\begin{tabular}{lll}
\hline Questions & Answers & Grade \\
\hline 1. How soon after you & Within 5 mins & 3 \\
wake up do you & $6-30$ mins & 2 \\
smoke your first & $31-60$ mins & 1 \\
$\quad$ cigarette? & More than 60 mins & 0 \\
\hline 2. Do you find it & Yes & 1 \\
difficult to refrain & & \\
$\quad$ from smoking in & No & 0 \\
$\quad$ places where it is & & \\
forbidden? & &
\end{tabular}

3. Which cigarette First one in morning 1

would you hate most

to give up? Other 0

4. How many cigarettes $\leq 10 \quad 0$

a day do you smoke? $11-20 \quad 1$

\begin{tabular}{cc}
$21-30$ & 2 \\
$>30$ & 3 \\
\hline
\end{tabular}

5. Do you smoke more Yes 1

frequently during

the first hours after

waking than during No 0

the rest of the day?

6. Do you smoke if you Yes

are so ill that you are

in bed most of the No 
day? 